\title{
Flexoelectricity in dielectrics: Materials, structures and characterizations*
}

\author{
Shujin Huang ${ }^{\dagger}$, Lu Qi ${ }^{\dagger} \ddagger$, Wenbin Huang ${ }^{\S}$, Longlong Shu ${ }^{\circledR}$, Shenjie Zhou ${ }^{\ddagger}$ \\ and Xiaoning Jiang ${ }^{\dagger}, \|$ \\ ${ }^{\dagger}$ Mechanial and Aerospace Engineering, \\ North Carolina State University, Raleigh, NC 27695, USA \\ ${ }^{\ddagger}$ School of Mechanical Engineering, Shandong University, \\ Jinan, Shandong 250100, P. R. China \\ ${ }^{\S}$ School of Mechanical Engineering, Chongqing University, \\ Chongqing 400044, P. R. China \\ \School of Materials Science and Engineering, \\ Nanchang University, Nanchang 330000, P. R. China \\ |xjiang5@ncsu.edu
}

Received 12 February 2018; Revised 28 March 2018; Accepted 29 March 2018; Published 7 May 2018

\begin{abstract}
Flexoelectricity in dielectrics suggests promising smart structures for sensors, actuators and transducers. In this review, dielectric materials, structures and the associated flexoelectric characterization methods are presented. First of all, we review structures and methods to measure different flexoelectric coefficients, including $\mu_{1122}, \mu_{1111}, \mu_{1211}, \mu_{3121}, \mu_{2312}, \mu_{1123}$, etc., via direct or converse flexoelectric effect. The flexoelectric materials in the form of bulk, thin films and 2D materials and the reported flexoelectric properties of these dielectrics will then be discussed. Semiconductor materials and the associated flexoelectric studies will also be reviewed. The progress of flexoelectric device study will next be presented, followed by the flexoelectricity research challenges and future trend.
\end{abstract}

Keywords: Flexoelectricity; dielectrics; semiconductor; thermal dependence; health monitoring.

\section{Principles of Flexoelectricity}

The rapid development in nanotechnology has satisfied human beings' fantasy of daily life. At the same time, it demands high-performance electronics, integrated circuits and micro/nanoelectromechanical systems. ${ }^{1}$ Electromechanical coupling effect, referring to the transformation from mechanical variables to electric variables or vice versa, underpins the functionality of an extremely broad range of materials and related devices. Currently, piezoelectricity is the main conversion mechanism between mechanical energy and electrical energy in those electromechanical devices, such as accelerometer, ${ }^{2}$ field effect transistors, ${ }^{3,4}$ self-powered nanogenerators, ${ }^{5-7}$ sensors and actuators. ${ }^{8}$ However, the materials selection is very limited due to the crystalline asymmetry constraint of piezoelectricity.

The flexoelectric effect describes the generation of an electric polarization response under a mechanical strain gradient (direct flexoelectric effect) or the mechanical response under an electric field gradient (converse flexoelectric effect). The existence of flexoelectricity in solid symmetric crystalline materials was originally discussed by Kogan in $1964 .^{9}$ As strain gradient can break the inversion symmetry, flexoelectricity allows the generation of electric polarization from lattice deformations in all dielectric materials. In mathematics, the flexoelectric effect is usually written in the form of

$$
\begin{aligned}
& P_{i}=\mu_{i j k l}^{\mathrm{dir}} \frac{\partial S_{j k}}{\partial x_{l}}, \quad P_{i}=f_{i j k l}^{\mathrm{dir}} \frac{\partial T_{j k}}{\partial x_{l}}, \\
& T_{i j}=\mu_{i j k l}^{\operatorname{con}} \frac{\partial E_{k}}{\partial x_{l}}, \quad S_{i j}=f_{i j k l}^{\text {con }} \frac{\partial E_{l}}{\partial x_{k}},
\end{aligned}
$$

where $P_{i}$ is the induced polarization, $T_{j k}$ is the stress, $S_{i j}$ is the induced strain and $E_{k}, E_{l}$ are the electric fields, $x$ is the axis of coordinate, $\mu_{i j k l}$ and $f_{i j k l}$ are the flexoelectric coefficients with respect to strain gradient and stress gradient, respectively. The superscripts "dir" and "con" indicate the direct and converse flexoelectric effects, respectively. The relationship between coefficients $\mu_{i j k l}$ and $f_{i j k l}$ can be deduced via the elastic constant:

$$
\mu_{i j k l}=c_{i j m n} \cdot f_{m n k l} .
$$

The concept of flexoelectricity was first originated from liquid crystals. ${ }^{10,11}$ The irregularly-shaped polarized molecules existing in liquid crystals will be reorientated by the mechanical strain gradient. Later, similar strain gradientinduced polarization phenomenon was also found in crystalline dielectrics, especially in the perovskites, as shown in Fig. 1. The $B$-site of the lattice will be deviated from the

\footnotetext{
*Selected paper from The International Workshop on Advanced Dielectric Materials and Their Applications, Xi'an, China, Oct. 8-11, 2017.
}

This is an Open Access article published by World Scientific Publishing Company. It is distributed under the terms of the Creative Commons Attribution 4.0 (CC-BY) License. Further distribution of this work is permitted, provided the original work is properly cited. 


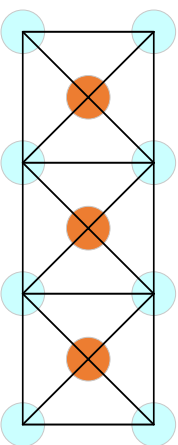

(a)

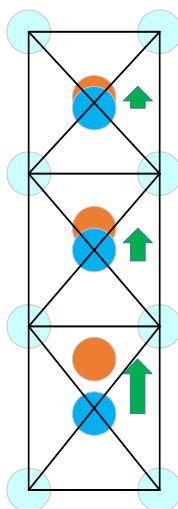

(b)

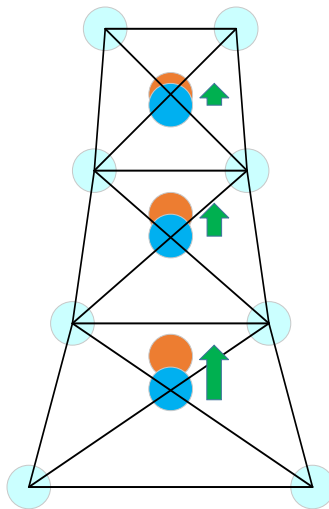

(c)
Cation

Anion
Flexoelectric polarization

Resulting negative charge
Fig. 1. Origin of flexoelectric effect in solids. (a) Two-dimensional (2D) structure of elementary charges without dipole moment. (b) Under uniformly tensile strain for each unit cell, the tension gradually varies from one cell to another. (c) Under inhomogeneous deformation, a dipole moment via the flexoelectric effect was induced within the unit cell. ${ }^{13}$

central position when the material is under an inhomogeneous strain. ${ }^{12}$

Theoretically, the flexoelectric effect in polymers such as polyvinylidene fluoride films (PVDFs) is similar to that in liquid crystals. However, the flexoelectric effect in polymer films is more complicated than that in solid crystals because both residual piezoelectricity effect and the flexoelectric effect should be considered. Thus the mechanism of flexoelectric effect in polymers has not been adequately understood so far.

In the nanoelectronic devices, strong strain gradients are often present, which imply the potential promising applications for flexoelectricity. ${ }^{14-16}$ Huang et al. showed that the flexoelectricity is a size-dependent effect due to the increasing of strain gradient when the size goes down. ${ }^{17}$

In this review, both experimental and theoretical studies will be included on the flexoelectricity in dielectrics, especially thermal effects on flexoelectricity. Furthermore, the flexoelectric responses of semiconductors are summarized and discussed. Furthermore, we summarize the current works on flexoelectric applications.

\section{Reported Experimental Studies and Theoretical Models $\left(\mu_{1122}, \mu_{1111}, \mu_{1211}, \mu_{3121}, \mu_{2312}\right.$ and $\left.\mu_{1123}\right)$}

To discover the materials with advanced flexoelectricity, it is essential to measure the flexoelectric coefficient of dielectrics, thin films, semiconductor materials and structures. In the last decades, experiments on a series of ferroelectrics have been performed due to their high dielectric constants as suggested by the lattice dynamic theory. ${ }^{12}$ For cubic crystals, mostly the ferroelectrics, only three independent components of the flexoelectric coefficient matrix are nonzero, which are $\mu_{1122}, \mu_{1111}$ and $\mu_{1211}$ (transverse, longitudinal and shear). For thin-film polymer, whose behavior is similar to liquid crystal, there are more nonzero components. In this section, we will summarize the experimental studies in two categories: direct flexoelectric measurement, through applying mechanical input and measuring the electrical polarization output; and converse flexoelectric measurement, where an external electrical voltage is applied to the materials and the mechanical output is monitored.

\subsection{Direct flexoelectric measurement}

Transverse and longitudinal flexoelectric coefficients ( $\mu_{1122}$ and $\left.\mu_{1111}\right)$ of dielectric perovskite ceramics were the first two components of the flexoelectric coefficient matrix being characterized. Cross and his co-worker first investigated $\mu_{1122}$ experimentally by using a cantilever system and a fourpoint bending system, shown in Fig. 2(a). ${ }^{18}$ Since then, various ferroelectric and paraelectric perovskite materials have been studied, such as barium titanate (BT), ${ }^{19}$ barium strontium titanate (BST), ${ }^{20}$ lead magnesium niobate (PMN) ${ }^{18,21}$ and lead zirconate titanate (PZT). ${ }^{22}$ Similar method has been adopted to study STO by Zubko et al. ${ }^{23}$ To eliminate the contributions of longitudinal and shear components, the sample was constrained to be a slender bar (length/width $>5$ ). In addition, due to the single-layer plate shape, the remnant piezoelectric contribution is symmetrically canceled.

$\mu_{1111}$ was studied by compressing a truncated pyramid specimen, shown in Fig. 2(b). ${ }^{24}$ This truncated pyramid shape was designed to generate longitudinal strain gradient when stress is applied. The average strain gradient in this truncated pyramid can be calculated from Eq. (3):

$$
\frac{\partial \varepsilon_{11}}{\partial x_{1}}=\frac{\Delta \varepsilon_{11}}{\Delta x_{1}}=\frac{\varepsilon_{11}^{u}-\varepsilon_{11}^{l}}{h} .
$$

Other than using continuous stress and strain, many other attempts on measuring the flexoelectric coefficient of ferroelectrics were made. The shock wave method succeeded in measuring the flexoelectric coefficient of bulk barium titanate. ${ }^{25}$

The shear flexoelectric coefficient $\left(\mu_{1211}\right)$ for bulk ceramic material has not been measured until very recently. It has been a technical barrier to generate a shear strain gradient on bulk ceramics. However it was easy to generate the shear strain gradient on polymers like PVDF. This material has been the one that was measured for the components of its flexoelectric coefficient matrix, including $\mu_{1211}, \mu_{3121}, \mu_{2312}$ and $\mu_{1123}{ }^{26-29}$

In addition to this shear component, the giant transverse and longitudinal flexoelectric coefficients in PVDF were observed. ${ }^{15,30-32}$ The difference apart from the flexoelectric characterization for solid material is that the residual piezoelectricity cannot be ignored for PVDF. The residual piezoelectricity is due to the residual electric polarization, 


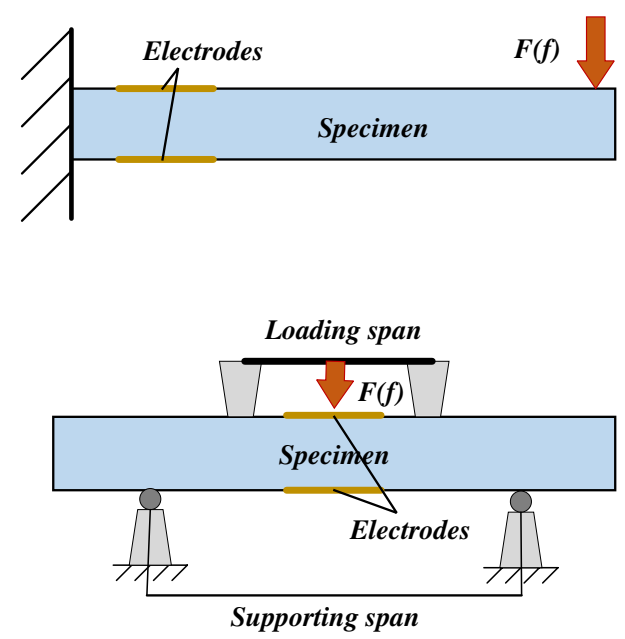

(a)

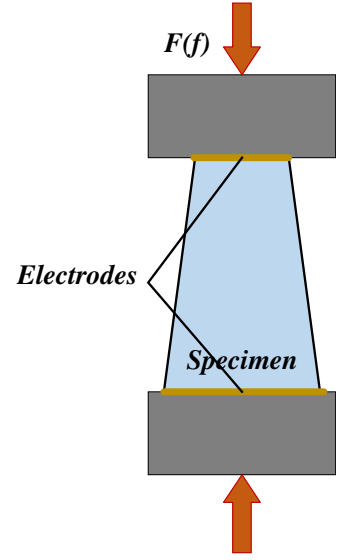

(b)

Fig. 2. The experimental setups for (a) $\mu_{1122}$ and (b) $\mu_{1111} \cdot{ }^{18,24}$

microstructural effects such as defects, cracks and the interaction between the $\alpha$-phase and the amorphous phase in the film.

\subsection{Converse flexoelectric measurement}

Comparing with the direct flexoelectric measurement, the converse flexoelectric measurement is more difficult to achieve mainly due to two critical issues. One is that the flexoelectric deformations have always been in the picometer scale for bulk ceramics, which is too small to detect. The other one is the simultaneous electrostriction effect. Unlike the piezoelectricity, the electrostriction effect has no symmetry constraint. The centrosymmetric materials are selected to exclude piezoelectricity but not the electrostriction effect. The electrostriction effect is a quadratic effect while the flexoelectric effect is a first-order effect. It is suggested that the electrostriction effect can be filtered out with a lock-in filter. However, Fu et al.'s initial experiments did not suggest so. ${ }^{33}$

To verify the converse flexoelectricity in BST ceramics, both longitudinal and lateral models were adopted. Fu et al. ${ }^{33}$ reported a longitudinal model by using an optical system to measure the displacement with the resolution as small as $10 \mathrm{E}-5 \mathrm{~nm}$. The optical system was based on the scanning Michelson laser beam interferometer, shown in Fig. 3.

There were two groups composed for the test: one was the flexoelectric group, where the electric field gradient existed; while the other was the control group where the electric field gradient was absent. As mentioned above, the flexoelectric group was expected to have first harmonic response, while only the second harmonic response should have appeared for the control group. But significant harmonic response appeared in the results for both the groups. The work was concluded as that the first harmonic response in samples of the control group was due to the first coupling of electrostriction effect with the DC electrical noise signal. The other work on converse flexoelectric effect was conducted on the

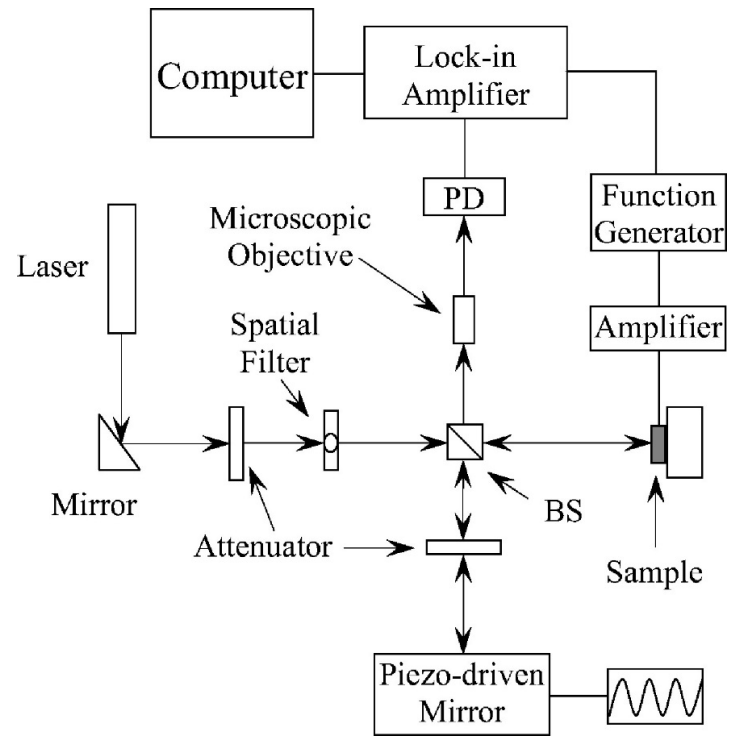

Fig. 3. An optical measurement system based on the scanning Michelson laser beam interferometer; PD: photodiode and BS: beam splitter.

Source: Reprinted with permission from Ref. 33.

$\mathrm{SrTiO}_{3}$ single crystal, ${ }^{34}$ which was structured as a cantilever beam. By applying a high-voltage $(0-3 \mathrm{kV})$ pulse for $2-3 \mathrm{~s}$, the bending curvature was measured by the deflection angle of the laser beam reflected from the surface of the unfixed edge. Instead of discussing the electrostriction effect, the temperature dependence of the effect within $77-450 \mathrm{~K}$ was obtained.

The lateral model was designed and reported by Shu et al. in order to isolate the converse flexoelectric effect from the electrostriction effect. ${ }^{35} \mathrm{~A}$ trapezoid BST block was used and the electrodes were attached to the lateral faces of this structure, where a nonparallel plate capacitor was created, as 


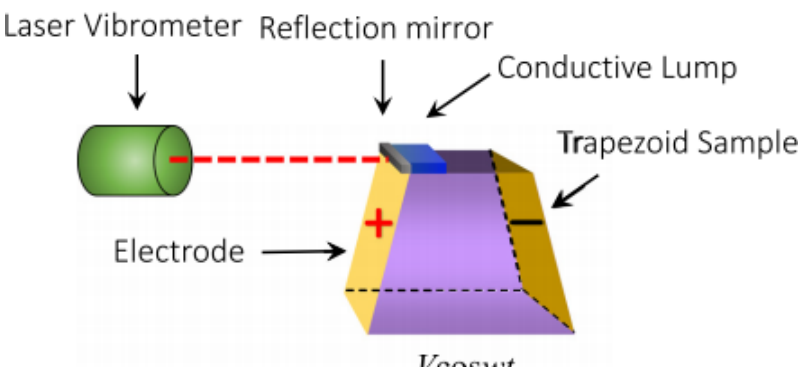

(a)

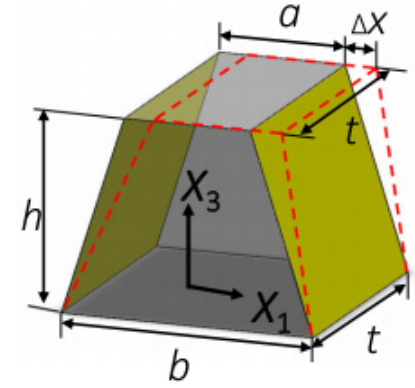

(b)

Fig. 4. Schematic for converse flexoelectric measurement of the shear strain along $x_{1}$-direction generated by the electric field gradient along the $x_{3}$-direction.

Source: Reprinted with permission from Ref. 35.

shown in Fig. 4. The expected result was that the electrostriction contribution should be canceled out because the product of the longitudinal electric field $\left(E_{1}\right)$ and the transverse electric field $\left(E_{3}\right)$ would be zero. However, the first harmonic response showed up in the results of both trapezoid and rectangle samples. By assuming all the coefficients for different shapes are the same, a calculated shear flexoelectric coefficient of $124 \pm 14 \mu \mathrm{C} / \mathrm{m}$ was reported.

Besides the experimental studies for converse flexoelectric measurement, Shu et al. intended to make the converse flexoelectric coefficient inclusive by studying the relation between the converse coefficient and the direct coefficient. ${ }^{36}$ Their theoretical derivation with Gibbs free energy density function suggested that, in crystalline mediums and systems, the direct and converse flexoelectric coefficients are equivalent. Due to the absence of practical experiments, this conclusion has assisted researchers to explore the applications of converse flexoelectric effects.

\subsection{Theoretical methods}

For flexoelectric (nonpiezoelectric and centrosymmetric) dielectrics, the most general bulk thermodynamic potential density $^{37-40}$ considering both polarization gradient and strain gradient is written as

$$
\begin{aligned}
U= & \frac{1}{2 \chi} P_{i} P_{i}+\frac{1}{2} b_{i j k l} P_{i, j} P_{k, l}+\frac{1}{2} c_{i j k l} \varepsilon_{i j} \varepsilon_{k l} \\
& +\frac{1}{2} g_{i j k l m n} \varepsilon_{i j, k} \varepsilon_{l m, n}+h_{i j k l} \varepsilon_{i j, k} P_{l} \\
& +k_{i j k l} \varepsilon_{i j} P_{l, k}-E_{i} P_{i}-\sigma_{i j} \varepsilon_{k l},
\end{aligned}
$$

where $\chi$ is the dielectric susceptibility. Also $c_{i j k l}, h_{i j k l}, k_{i j k l}$, $b_{i j k l}$ and $g_{i j k l m n}$ are the material parameter tensors. $P_{i}, P_{i, j}$ denote the polarization and polarization gradient tensors, respectively. $E_{i}$ denotes the electric field and $\sigma_{i j}$ is the stress tensor. $\varepsilon_{i j}, \varepsilon_{i j, k}$ are the strain and strain gradient tensors, respectively, defined as

$$
\varepsilon_{i j}=\frac{1}{2}\left(u_{i, j}+u_{j, i}\right) .
$$

Here, $u_{i}$ represents the displacement vector and the comma in the subscript indicates differentiation with respect to the spatial variables, i.e., $(\cdots)_{, i}=\partial(\cdots) / \partial x_{i}$.

In the bulk thermodynamic potential density, the terms $\frac{1}{2} b_{i j k l} P_{i, j} P_{k, l}$ and $\frac{1}{2} g_{i j k l m n} \varepsilon_{i j, k} \varepsilon_{l m, n}$, respectively, represent the pure polarization and strain gradients effects, which are not included in the classical piezoelectric theory. The term $h_{i j k l}$ $\varepsilon_{i j, k} P_{l}+k_{i j k l} \varepsilon_{i j} P_{l, k}$ describes the flexoelectric coupling effect, which can be expressed in other forms using the integration by parts. Here, the most used three other forms ${ }^{41-43}$ are

$$
\begin{aligned}
& h_{i j k l} \varepsilon_{i j, k} P_{l}+k_{i j k l} \varepsilon_{i j} P_{l, k} \\
& \quad=\frac{h_{i j k l}-k_{i j k l}}{2}\left(\eta_{i j k} P_{l}-\varepsilon_{i j} P_{l, k}\right)+\frac{h_{i j k l}+k_{i j k l}}{2}\left(\varepsilon_{i j} P_{l}\right)_{, k} \\
& \quad=\left(h_{i j k l}-k_{i j k l}\right) \eta_{i j k} P_{l}+k_{i j k l}\left(\varepsilon_{i j} P_{l}\right)_{, k} \\
& \quad=\left(k_{i j k l}-h_{i j k l}\right) \varepsilon_{i j} P_{l, k}+h_{i j k l}\left(\varepsilon_{i j} P_{l}\right)_{, k} .
\end{aligned}
$$

In each form, the last term will be treated as a surface term because the whole derivative cannot be included in the bulk energy density. These expressions lead to the same governing equations but with different natural boundary conditions.

Applying the Euler equations $\partial H / \partial A-\left(\partial H / \partial A_{, i}\right)_{, i}=0$, where $A$ stands for $P_{i}$ or $\varepsilon_{i j}$, the bulk constitutive electromechanical equations will be obtained:

$$
\begin{gathered}
P_{i}-\chi b_{i j k l} P_{k, l j}=\chi E_{i}+\chi\left(k_{k j l i}-h_{k j l i}\right) \varepsilon_{j k, l}, \\
\sigma_{i j}=c_{i j k l} \varepsilon_{k l}+\left(k_{i j k l}-h_{i j k l}\right) P_{l, k}-g_{i j k l m n} \varepsilon_{l m, n k} .
\end{gathered}
$$

In Eq. (7), the first term in the right-hand side describes the classical dielectric response to an electric field. The second term describes the direct flexoelectric response. In Eq. (8), the first term in the right-hand side describes the classical Hook's law and the second term describes the converse flexoelectric response. In addition, it should be noted that $\chi b_{i j k l} P_{k, l j}$ and $g_{i j k l m n} \varepsilon_{l m, n k}$, which are derived from the pure polarization and strain gradients terms, respectively, represent the higher-order effects. These higher-order effects could influence the polarization field and the stress field in the nanoscale or even in the microscale provided the characterized size of the structure (for example, the thickness of a 
dielectric cantilever beam) is comparable to the effective range of these effects. ${ }^{44-46}$ Unfortunately, the coefficients of these higher-order terms are scarcely quantified in most dielectrics, which makes it difficult to verify the effective range of these higher-order effects. Generally, these higherorder terms are neglected especially in the experiment (the characteristic size is tens or hundreds of microns). Substituting Eq. (7) into Eq. (5) and neglecting the higher-order effects, we find the most general constitutive equations characterizing the flexoelectric effects:

$$
\begin{aligned}
& P_{i}=\chi E_{i}+\mu_{i j k l} \varepsilon_{j k, l}, \\
& \sigma_{i j}=c_{i j k l} \varepsilon_{k l}+\mu_{l j i k} E_{l, k} .
\end{aligned}
$$

Here, $\mu_{i j k l}=\chi\left(k_{k j l i}-h_{k j l i}\right)$ is the flexoelectric coefficient. The tensor $\left(k_{k j l i}-h_{k j l i}\right)$ is called the flexoelectric coupling coefficient that can be represented by an independent tensor $f_{i j k l}$. It is obvious that the flexoelectric coefficient is proportional to the relative dielectric constants and the flexoelectric coupling coefficients, the values of which were theoretically estimated by Kogan, ${ }^{9}$ Tagantsev $^{12}$ and Hong and Vanderbilt $\mathrm{t}^{47,48}$ to be around several tens of volts for simple ideal ionic solids. These may be called the intrinsic (or lattice-based) flexoelectric coupling coefficients. However, more and more experimental results showed that the apparent (or effective) flexoelectric coupling coefficients in many dielectrics can reach up to several hundred or even several thousand volts. ${ }^{17,19,49}$ Therefore, it appears there are other dominant origins contributing to the apparent flexoelectric responses.

Many researchers have done a lot of work to investigate the anomaly-enhanced flexoelectric responses and some possible origins were proposed. ${ }^{23,50-56}$ First, the initial strain gradients formed around the interface, grain boundary or defects have been reported to possibly enhance the flexoelectric responses. These local strain gradients can reach up to as large as $10^{6}-10^{7} \mathrm{~m}^{-1}$, which are $7-8$ orders of magnitude larger than those reported for bulk solids. For example, using grazing-incidence in-plane X-ray diffraction, Lee et al. measured the strain gradient, induced by a strain relaxation with tens of nanometers of the film-substrate interface due to the lattice mismatch, to be $10^{5}-10^{6} \mathrm{~m}^{-1} \cdot{ }^{51}$ Zubko et al. believed the strain gradient near the defect can be as large as $10^{7} \mathrm{~m}^{-1} \cdot{ }^{23}$ The extremely large strain gradients will induce large local polarization and should contribute to the effective flexoelectric responses. Another main origin of the anomalously large effective flexoelectric responses may come from the residual ferroelectricity, of which the polar nanoregion is one important source. Narvaez and Catalan found that the ferroelastic switching induced by the stress combined with the intrinsic flexoelectric field may pole the relaxor ferroelectrics such as PMN-PT even at the room temperature. ${ }^{50}$ Similar enhancement of apparent flexoelectric responses due to the residual ferroelectricity is also found in the BST ceramics by Garten et al. ${ }^{52}$ and much recently in
$0.3 \mathrm{~Pb}\left(\mathrm{In}_{1 / 2} \mathrm{Nb}_{1 / 2}\right) \mathrm{O}_{3}-0.35 \mathrm{~Pb}\left(\mathrm{Mg}_{1 / 3} \mathrm{Nb}_{2 / 3}\right) \mathrm{O}_{3}-0.35 \mathrm{PbTiO}_{3}$ (PIN-PMT-PT) by Shu et al. ${ }^{54}$

All in all, due to the complex microscale or nanoscale structures existing in the dielectrics, the effective flexoelectric responses may be mixed effects of many factors such as the surface piezoelectricity, intrinsic piezoelectricity, residual ferroelectricity and defects, which still need to be verified in terms of both theory and experiment.

\section{Flexoelectricity of Dielectrics}

\subsection{Bulk material}

Both experimental studies and theoretical derivation have suggested that the enhanced flexoelectricity may possibly exist when the materials have a high dielectric permittivity. All measured flexoelectric coefficients up to date are summarized in Table 1, along with their dielectric permittivity values. It is worth mentioning that more different topographic structures of dielectric materials were not studied to measure the longitudinal or shear flexoelectric component.

Instead, the temperature dependence has caught the attention. It has been reported that flexoelectricity is positively correlated with the dielectric constant in solid crystalline materials. $^{24}$ The temperature dependence of flexoelectric coefficients has been investigated since the dielectric permittivity has a peak value at the Curie temperature. Furthermore, comparing to the piezoelectric materials, it is expected that flexoelectric materials can work in a broader temperature. It was found in BST that the flexoelectric coefficient approaches its peak at the phase transition point. ${ }^{24}$ Later on, the temperature dependence of flexoelectricity in $\mathrm{BaTiO}_{3}-$ $0.08 \mathrm{Bi}\left(\mathrm{Zn}_{1 / 2} \mathrm{Ti}_{1 / 2}\right) \mathrm{O}_{3}$ (BT-8BZT), BaTiO ${ }_{3}$ and $\mathrm{SrTiO}_{3}$ perovskite nanostructures were also measured. ${ }^{58,59}$ For BST, $\mathrm{BaTiO}_{3}$ and $\mathrm{SrTiO}_{3}$, the rapid drop in the dielectric permittivity in the paraelectric phase leads to an inconsistent flexoelectricity as temperature increases. The narrow and sharp dielectric peak occurring at the ferroelectric-to-paraelectric phase transition limits the enhanced flexoelectric effect within a narrow temperature range. All these experiments characterized the flexoelectricity under $70^{\circ} \mathrm{C}$, which are not qualified for the high-temperature application.

Thus, to find a material whose dielectric permittivity would smoothly decline would be the direction of material

Table 1. Measured flexoelectric coefficient values and the dielectric permittivities of dielectric materials (at room temperature).

\begin{tabular}{lcccc}
\hline Material & $\varepsilon_{r}$ & $\mu_{1122}(\mu \mathrm{C} / \mathrm{m})$ & $\mu_{1111}(\mu \mathrm{C} / \mathrm{m})$ & $\mu_{1212}(\mu \mathrm{C} / \mathrm{m})$ \\
\hline PMN & 11,720 & $3.4^{18}$ & & \\
PZT & 2130 & $1.4^{22}$ & & \\
BST & 13,200 & $20-100^{17,20}$ & $120^{33}$ & $110\left(f_{1212}\right)^{35}$ \\
BT & 2300 & $9^{19}$ & & $3 \mathrm{E}-3^{23}$ \\
STO & 300 & $4 \mathrm{E}-3^{23}$ & $9 \mathrm{E}-3^{23}$ & \\
PMN-PT & $\sim 5000$ & $70.8-100.8^{57}$ & & \\
\hline
\end{tabular}


hunting. The gradual decline of flexoelectric coefficient $\mu_{12}$ with increasing temperature in BT-8BZT was reported by Huang et al. ${ }^{59}$ The temperature range goes beyond $200^{\circ} \mathrm{C}$. The measured flexoelectric coefficient $\mu_{12}$ of BT$8 \mathrm{BZT}$ is $25 \mu \mathrm{C} / \mathrm{m}$ at $25^{\circ} \mathrm{C}$ and can remain above $12 \mu \mathrm{C} / \mathrm{m}$ at temperatures up to $200^{\circ} \mathrm{C}$. This suggests a new group of compositions in the $\mathrm{BiMeO}_{3}-\mathrm{BaTiO}_{3}$ system to be studied for its flexoelectricity, since these compositions exhibit temperature-stable permittivity over a much wider range. ${ }^{60}$

On the other hand, a high Curie temperature material can also be considered for flexoelectricity. Very recently, Shu et al. have reported the flexoelectric behavior in PIN-PMTPT single crystal. ${ }^{54}$ The Curie temperature of PIN-PMT-PT is at $229^{\circ} \mathrm{C}$ but the crystal undergoes a macrodomain-tomicrodomain transformation at $209^{\circ} \mathrm{C}$. The maximum effective flexoelectric coefficient is $125 \mu \mathrm{C} / \mathrm{m}$ measured at $209^{\circ} \mathrm{C}$. But above Curie temperature, it decrease to $27 \mu \mathrm{C} / \mathrm{m}$.

Although significant advances of flexoelectricity have been made in the past decades, the origin of the enhanced flexoelectricity has not been fully understood. In ceramics, interference factors, such as the grain boundary, were discussed because of their influence on the flexoelectricity. ${ }^{61}$ Single crystals, on the other hand, could minimize such effects. But fewer studies on single crystals were reported. Whether flexoelectricity is a bulk effect or a surface effect has been in debate. Catalan and co-workers' recent work on $\mathrm{BaTiO}_{3}$ single crystal has emphasized the flexoelectricity with enlarged residual surface piezoelectricity. ${ }^{62}$ Shu et al. discovered the frequency dispersion phenomenon in PMNPT at a relatively low frequency. ${ }^{57}$ This dispersion occurred with a time delay but had a positive relationship with frequency. Thus it is proved that the flexoelectric effect acts more like a bulk effect rather than a surface effect. Meanwhile, it was revealed that for the single crystal, the frequency is highly related to the crystal structure, and it is easier to enhance by the ferroelectricity.

\subsection{Thin films}

Because the strain gradient is always inversely proportional to the material size, the induced flexoelectricity by strain gradient is expected to be larger in thin films compared with bulk materials. Due to large flexoelectric coefficient of BST bulk ceramics, the flexoelectricity in BST thin film was studied. ${ }^{63}$ Also, from the principle, if the deformation applied to one structure is larger, the strain gradient would be larger. ${ }^{64}$ Then the flexoelectric response is stronger. Polymer thin films, especially PVDF, have their advantages in this aspect. All the reported components of the flexoelectric coefficient matrix are listed in Table 2.

\subsection{Two-dimensional crystalline membrane}

As mentioned, flexoelectricity is strongly scale-dependent. Compared to thin film, 2D crystalline membranes are nearly
Table 2. Flexoelectric coefficient matrix components of PVDF.

\begin{tabular}{lcccc}
\hline $\begin{array}{l}\mu_{1122} \\
(\mu \mathrm{C} / \mathrm{m})\end{array}$ & $\begin{array}{c}\mu_{1111} \\
(\mu \mathrm{C} / \mathrm{m})\end{array}$ & $\begin{array}{c}\mu_{1211} \\
(\mu \mathrm{C} / \mathrm{m})\end{array}$ & $\begin{array}{c}\mu_{3121} \\
(\mu \mathrm{C} / \mathrm{m})\end{array}$ & $\begin{array}{c}\mu_{1123} \\
(\mu \mathrm{C} / \mathrm{m})\end{array}$ \\
\hline $34.5,37.3^{31}$ & $\begin{array}{c}-78.19, \\
1.6 \mathrm{E}-2^{65,66}\end{array}$ & $7.318 \mathrm{E}-4^{26}$ & $1.037 \mathrm{E}-2^{27}$ & $(9.2-9.6) \mathrm{E}-3^{29}$ \\
\hline
\end{tabular}

atomistically thin. Though the flexoelectricity is negligibly small for many dielectrics on the conventional scale, it becomes dominant at the nanoscale. The scaling prediction was first proposed by Majdoub et al. and recently confirmed by various experimental evidences. ${ }^{14}$ Those experimental evidences indicated that the flexoelectricity has a promising application potential in charge transportation, domain engineering, random access memory and nanoscale actuating device. $^{67}$ For membranes, flexoelectric polarization can be simplified down to a simple proportional relation with the mean curvature of the membrane in the direction normal to the membrane. Similar to a thin paper-like structure, it is easy to induce a strain gradient by bending, so the researchers have focused on its potential applications. There have been rapid computational developments but rarely reported experimental results. Piezoelectricity of such materials was first explored for an enhanced electromechanical coupling performance. Graphene nitride nanosheet, riddled by triangular holes, was experimentally and computationally shown to exhibit an apparent piezoelectric response. ${ }^{68}$ Piezoelectricity in materials such as $\mathrm{BN}, \mathrm{MoS}_{2}$ and $\mathrm{WS}_{2}$ was calculated as well. ${ }^{67,69}$ The characterization data of flexoelectricity in these twodimensional materials are yet missing.

Sharma et al. have published a thorough review on this topic including the biological membrane recently and their focus was primarily on mathematical and computational developments. They pointed out that the flexoelectricity has a number of implications in biology including ion transport, electromotility and mammalian hearing mechanism. ${ }^{67,73}$ They also summarized experimental findings in organic materials such as lipid bilayers. ${ }^{73}$

\subsection{Semiconductors and their flexo-like response}

Although the flexoelectricity has always been studied in dielectric insulators, the insulation is not a formal requirement for the property. Theoretically, the polarization induced by strain gradients would be responsible for the redistribution of the free charge in semiconductors.

Catalan and co-workers reported the significantly enhanced flexoelectricity in vacuum-annealed $\mathrm{BaTiO}_{3}$ semiconductor, which was attributed to a barrier layer mechanism. ${ }^{62}$ The mechanism of enhanced flexoelectricity in semiconductive, treated BTO single crystals is shown in Fig. 5. As reported, the effective flexoelectric coefficient reached $1 \mathrm{mC} / \mathrm{m}$, increased by orders of magnitude since all conventional flexoelectric coefficients were reported in the order of $\mu \mathrm{C} / \mathrm{m}$. 
(a)
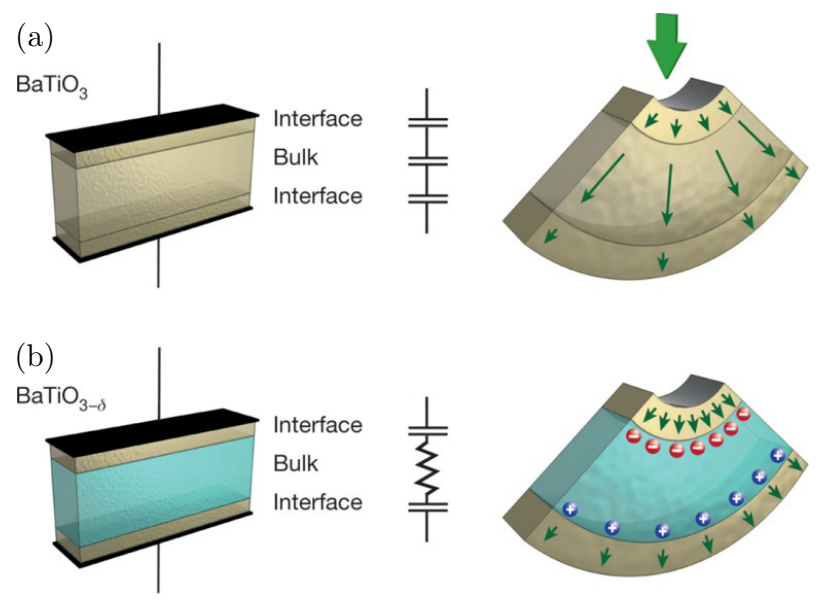
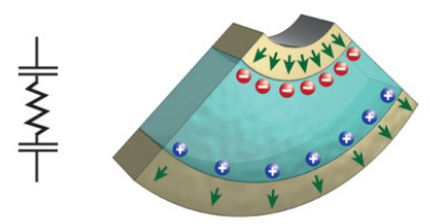

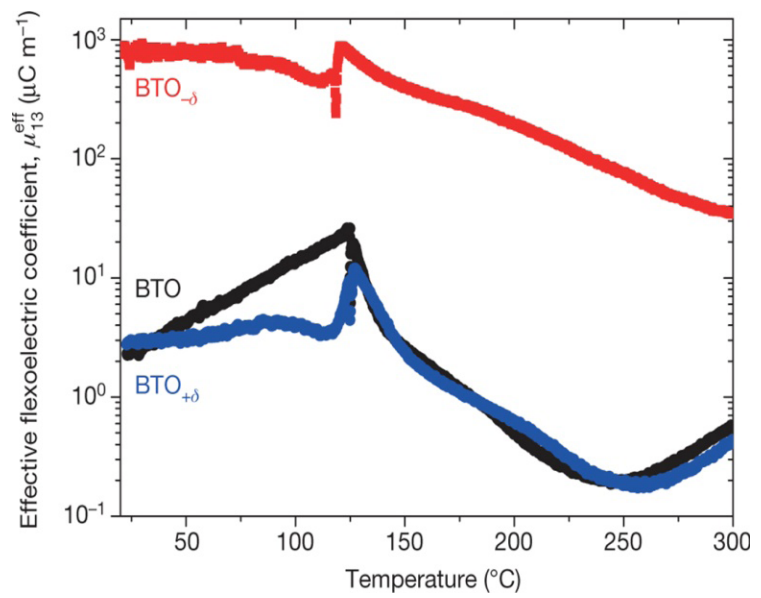

Fig. 5. Effective flexoelectric behavior of semiconductive $\mathrm{BaTiO}_{3}$.

Source: Reprinted with permission from Ref. 62.

Serry and Sakr have reported a semiconductor structure with graphene-metal-silicone for multimodal energy conversion. ${ }^{74}$ Flexoelectric behavior can be observed and further modulated in the fabrication of composite structures. During the fabrication, a mismatch occurred in the mechanical and thermal properties between different layers. At the same time, residual stresses exist at the interface of the structural layers induced from the thin-film deposition processes, which were tested through pure bending and thermomechanical tests. Therefore mechanical and thermal energy conversions are achieved through both flexoelectric and thermionic effects.

\section{Applications}

Although the studies of flexoelectricity in materials have been proceeding, the flexoelectric effect is still not as large as the piezoelectric effect. Designing new flexoelectric materials has become a fundamental issue in this newly arising field. Engineering the current materials to obtain great flexoelectric coefficient has become one trend to satisfy the standard of real applications, especially in passive sensing devices. Meanwhile, people started to introduce flexoelectricity into energy harvesting devices and structural health monitoring (SHM) sensors. In this section, the works in these two branches will be summarized.

\subsection{Materials design}

The generation of strain gradients should come from the external deformation of the material or the internal stress of the materials. Characterization techniques always introduce external force into the materials in order to observe large deformation. The internal stress of the material has rarely been engineered. Chu and co-workers have demonstrated a material type named as flexoelectric/piezoelectric metamaterials by using NBBT, PZT-51, PZT-81 and BST ceramics. $^{75-78}$ The fabrication method was asymmetric reduction, which was adopted from the fabrication of RAINBOW and THUNDER piezoelectric materials. Because of the reduction-induced chemical inhomogeneity, a strain gradient was generated so that the apparent electromechanical coupling effect was enhanced. As a result, its effective piezoelectric coefficients approached $57 \mathrm{pC} / \mathrm{N}$ at the temperature near Curie temperature, where the nominal piezoelectric device would become nonexistent.

On the other hand, design of composites using the currently known flexoelectric material has been undergoing. Chu et al. initially proposed a two-dimensional flexure composite model with BST sheets and tungsten balls. ${ }^{79}$ Due to the existence of strain gradient, a giant electric polarization was exhibited in this composite and the effective piezoelectric coefficients were calculated to exceed $4300 \mathrm{pC} / \mathrm{N}$. Shu and co-workers recently followed this model and systematically designed a flexure composite consisting of BST ceramic and the supporting material based on a parametric sweep finite element method (FEM) simulation. The design parameters are shown in Fig. $6 .^{80}$ The optimized composite structure can enhance the performance of the initial model up to 50 times by FEM simulation, with the thickness/length ratio of BST at $1 / 150$, and with silicon carbide as the supporting material at $2.5 \mathrm{~mm}$ distance from the top and $2.1 \mathrm{~mm}$ distance from the bottom.

\subsection{Devices: Sensors, energy harvester and microphone}

Previously, in 2013, the potentials of nanogenerators were summarized and discussed. ${ }^{81}$ Since then, there have been some investigations on materials which exhibit high flexoelectric coefficients as we mentioned previously. However, the proper degradation of nanoscale structures is still needed for inventing flexoelectric devices. Up to now, flexoelectric devices have been designed mainly in two application 


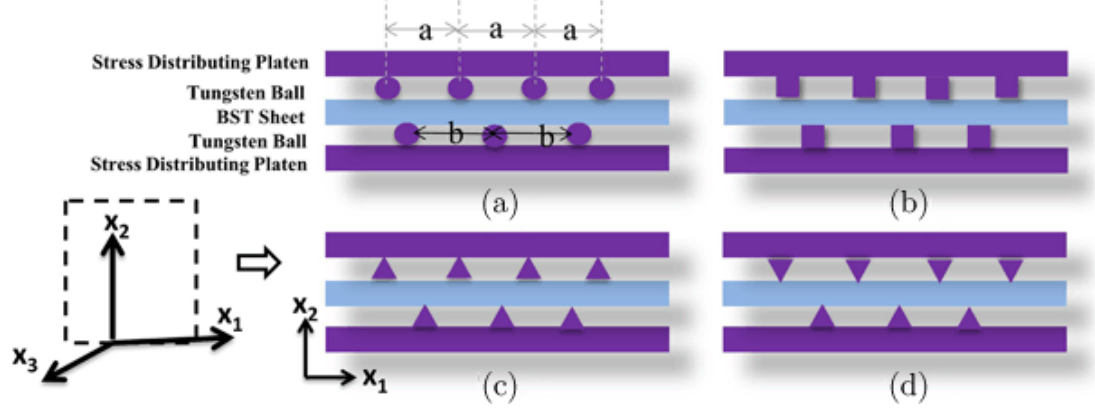

Fig. 6. Schematic of the flexure composite with geometry varied supporting material.

Source: Reprinted with permission from Ref. 78.

directions, actuators and sensors, like nominal electromechanical converters by piezoelectricity.

Structural health monitoring in mechanical, architectural and transport vehicles has been the key area for flexoelectric detections. In such systems, defects like cracks would cause catastrophic accidents. Because of the scaling effect of gradient, the sensitivity and accuracy of flexoelectric sensors could stand out among existing detection technologies. Huang and co-workers published the flexoelectric strain gradient sensor for structural health monitoring and crack

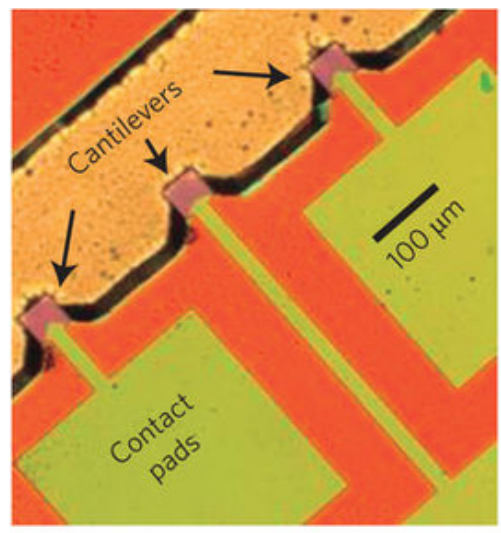

(a)
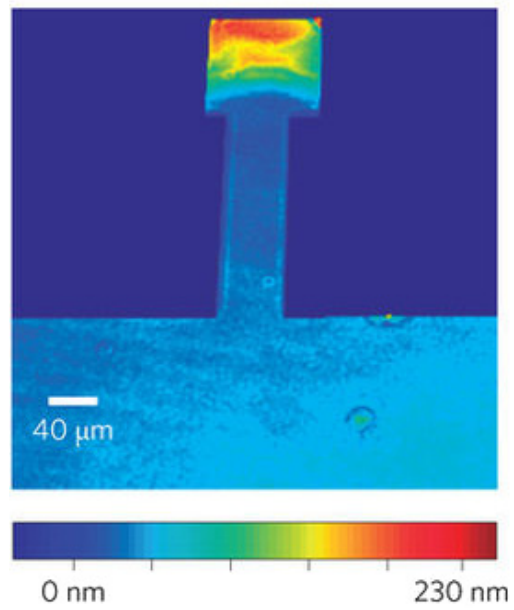

(b)

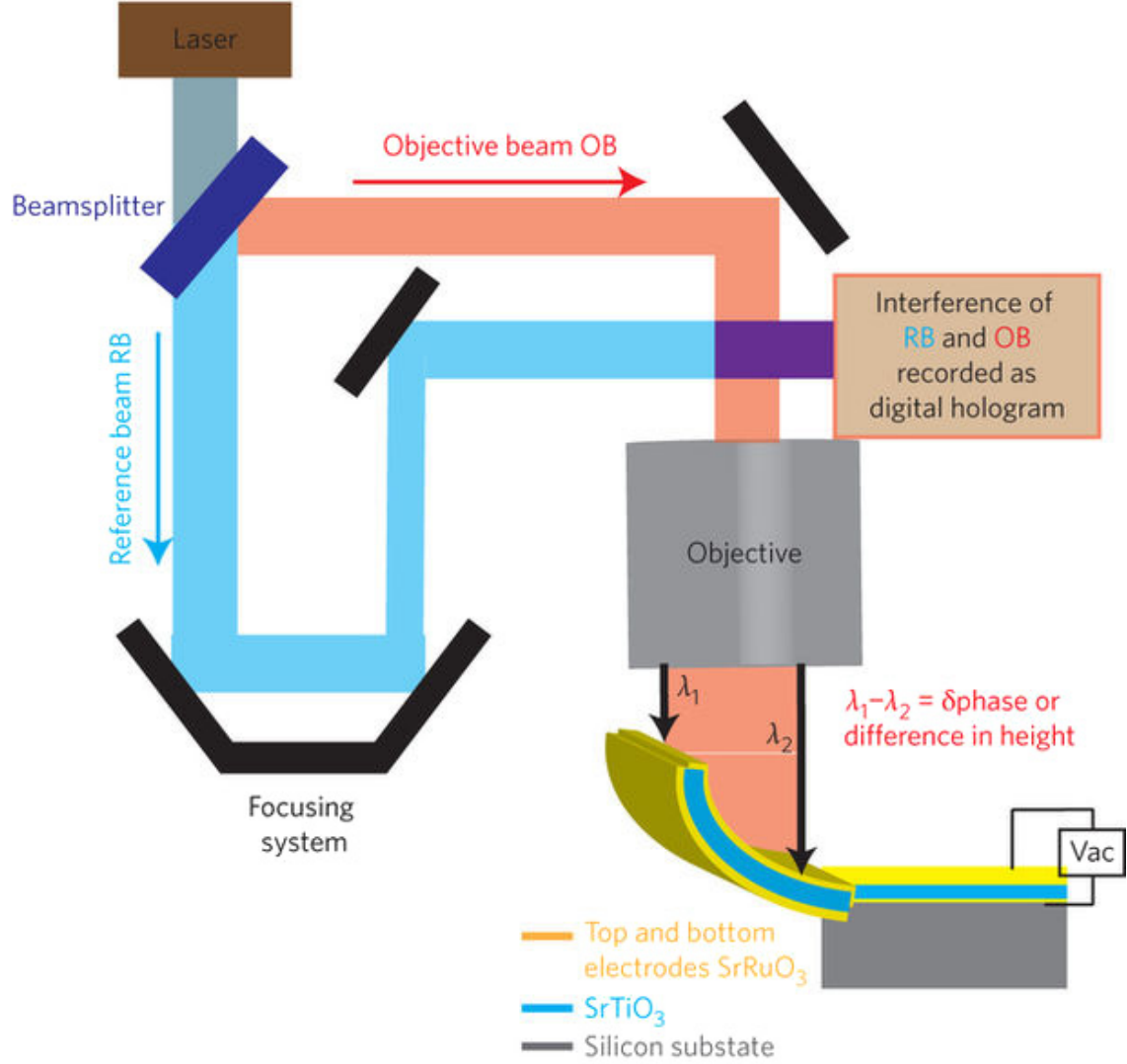

(c)

Fig. 7. (a) Optical image of an array of $\mathrm{SrTiO}_{3}$ nanocantilevers. (b) Three-dimensional image of one $\mathrm{SrTiO}_{3}$ nanocantilever with color scale corresponding to the out-of-plane displacement. (c) The digital holographic microscope experiment setup.

Source: Reprinted with permission from Ref. 90. 
detection. ${ }^{82-85}$ Due to the stress concentration at the surrounding of a crack, the strain gradient varies abruptly in the vicinity of the crack. The sensor was attached to the neighborhood of a cracking site. With varied tension stress, the induced charge due to flexoelectricity was measured to predict the cracking site. Tzou and co-workers have reported a distributed system for structural sensing by flexoelectricity. ${ }^{86}$ A group of BST ceramic sensors were distributed on a cantilever flexoelectric beam and their flexoelectric constants were found consistent by the open-circuit model. An alternative for dynamic measurements of mechanical structures was provided.

The performance of micro/nanofabricated sensors can also be improved by adopting the flexoelectric effect. Kwon et al. first designed and fabricated a highly sensitive flexoelectric microphone, which can work at an extremely wide working frequency range based on barium strontium titanate ceramic. $^{87}$

As for energy harvesting, Zhang et al. reported a curved resonant flexoelectric actuator fabricated with PVDF. ${ }^{88}$ Tzou and co-workers claimed the static nanocontrol beams. ${ }^{89}$ Catalan and co-workers fabricated a pioneer cantilever actuator with strontium titanate integrated onto silicon, shown in Fig. $7 .{ }^{90}$ A figure of merit (curvature divided by electric field) of $3.33 \mathrm{MV}^{-1}$ was reported to be comparable to that of state-of-the-art piezoelectric bimorph cantilever. Similar structure was also made with PZT which showed the flexoelectricity can either enhance or suppress the piezoelectric response of the cantilever depending on the ferroelectric polarity and lead to a diode-asymmetric electromechanical response.

Besides all above known areas where flexoelectricity can show its advantages, thermal gradient-induced flexoelectric effects were first studied by Jiang and co-workers in bulk BST ceramics. $^{91}$ Wave propagation within the microstructured solid was observed by Shen's group and Wei's group. ${ }^{92,93}$ These new applications have truly broadened the research on flexoelectricity.

\section{Future Work and Challenges}

So far, in the research field of flexoelectricity, researchers had still mainly focused on bulk dielectrics and some thin films. Only few findings have been reported in other material categories. How to further optimize the flexoelectric performance and how to design flexoelectric devices have become critical in this newly arising field. Nanoscale materials like 2D materials and some semiconductors may be advanced in terms of flexoelectricity. It may lead to an edge that flexoelectric effect would go beyond the conventional piezoelectric effect due to its scale and its thermal advantages. On one hand, the studies of its origin and the characterization of flexoelectric coefficient matrix in bulk dielectric are not convincing and completed. On the other hand, the potential applications in sensing and actuating devices urge developing a classical flexoelectric material that is excluded from the piezoelectric materials. The composite design and the material search should keep going. Furthermore, more energy harvesting devices made with current known materials with high flexoelectricity should be designed and tested. The scaling down trend of miniature devices or the lab-on-chip industry needs new type of electromechanical devices that can be easily integrated with other elements on the chip at the nanoscale.

\section{References}

${ }^{1}$ H. G. Craighead, Nanoelectromechanical systems, Science 290, 1532 (2000).

${ }^{2}$ J.-C. Yu and C.-B. Lan, System modeling of microaccelerometer using piezoelectric thin films, Sens. Actuators A, Phys. 88, 178 (2001).

${ }^{3}$ Y. Nishi, Field effect transistors (1978).

${ }^{4}$ A. Javey, J. Guo, Q. Wang, M. Lundstrom and H. Dai, Ballistic carbon nanotube field-effect transistors, Nature 424, 654 (2003).

${ }^{5}$ Z. L. Wang, Towards self-powered nanosystems: From nanogenerators to nanopiezotronics, Adv. Funct. Mater. 18, 3553 (2008).

${ }^{6} \mathrm{~S}$. Xu et al., Self-powered nanowire devices, Nat. Nanotechnol. 5 , 366 (2010).

${ }^{7}$ F.-R. Fan et al., Transparent triboelectric nanogenerators and selfpowered pressure sensors based on micropatterned plastic films, Nano Lett. 12, 3109 (2012).

${ }^{8}$ S. K. Park and X. L. Gao, Bernoulli-Euler beam model based on a modified couple stress theory, J. Micromech. Microeng. 16, 2355 (2006).

${ }^{9}$ S. M. Kogan, Piezoelectric effect during inhomogeneous deformation and acoustic scattering of carriers in crystals, Sov. Phys.Solid State 5, 2069 (1964).

${ }^{10}$ R. E. Newnham, Properties of Materials: Anisotropy, Symmetry, Structure (Oxford University Press on Demand, 2005).

${ }^{11}$ P. J. Olver, Applications of Lie Groups to Differential Equations, Vol. 107 (Springer Science \& Business Media, 2000).

${ }^{12}$ A. K. Tagantsev, Piezoelectricity and flexoelectricity in crystalline dielectrics, Phys. Rev. B 34, 5883 (1986).

${ }^{13}$ S. V. Kalinin and A. N. Morozovska, Multiferroics: Focusing light on flexoelectricity, Nat. Nanotechnol. 10, 916 (2015).

${ }^{14}$ M. S. Majdoub, R. Maranganti and P. Sharma, Understanding the origins of the intrinsic dead layer effect in nanocapacitors, Phys. Rev. B 79, 115412 (2009).

${ }^{15} \mathrm{~S}$. Baskaran, X. He and J. Y. Fu, Gradient scaling phenomenon of piezoelectricity in non-piezoelectric polyvinylidene fluoride films, arXiv Prepr. arXiv1110.5093 (2011).

${ }^{16}$ D. Lee, S. M. Yang, J.-G. Yoon and T. W. Noh, Flexoelectric rectification of charge transport in strain-graded dielectrics, Nano Lett. 12, 6436 (2012).

${ }^{17}$ W. Huang, K. Kim, S. Zhang, F. Yuan and X. Jiang, Scaling effect of flexoelectric (Ba, Sr) $\mathrm{TiO}_{3}$ microcantilevers, Phys. Status Solidi (RRL)-Rapid Res. Lett. 5, 350 (2011).

${ }^{18}$ W. Ma and L. E. Cross, Large flexoelectric polarization in ceramic lead magnesium niobate, Appl. Phys. Lett. 79, 4420 (2001).

${ }^{19}$ W. Ma and L. E. Cross, Flexoelectricity of barium titanate, Appl. Phys. Lett. 88, 2902 (2006).

${ }^{20} \mathrm{~W}$. Ma and L. E. Cross, Flexoelectric polarization of barium strontium titanate in the paraelectric state, Appl. Phys. Lett. 81, 3440 (2002) 
${ }^{21}$ W. Ma and L. E. Cross, Observation of the flexoelectric effect in relaxor $\mathrm{Pb}\left(\mathrm{Mg}_{1 / 3} \mathrm{Nb}_{2 / 3}\right) \mathrm{O}_{3}$ ceramics, Appl. Phys. Lett. 78, 2920 (2001).

${ }^{22}$ W. Ma and L. E. Cross, Flexoelectric effect in ceramic lead zirconate titanate, Appl. Phys. Lett. 86, 72905 (2005).

${ }^{23}$ P. Zubko, G. Catalan, A. Buckley, P. R. L. Welche and J. F. Scott, Strain-gradient-induced polarization in $\mathrm{SrTiO}_{3}$ single crystals, Phys. Rev. Lett. 99, 167601 (2007).

${ }^{24}$ L. E. Cross, Flexoelectric effects: Charge separation in insulating solids subjected to elastic strain gradients, J. Mater. Sci. 41, 53 (2006).

${ }^{25}$ T. Hu, Q. Deng, X. Liang and S. Shen, Measuring the flexoelectric coefficient of bulk barium titanate from a shock wave experiment, J. Appl. Phys. 122, 55106 (2017).

${ }^{26}$ S. Zhang, M. Xu, X. Liang and S. Shen, Shear flexoelectric coefficient $\mu 1211$ in polyvinylidene fluoride, J. Appl. Phys. 117, 204102 (2015).

${ }^{27}$ S. Zhang, X. Liang, M. Xu, B. Feng and S. Shen, Shear flexoelectric response along 3121 direction in polyvinylidene fluoride, Appl. Phys. Lett. 107, 142902 (2015).

${ }^{28} \mathrm{~S}$. Zhang et al., Investigation of the 2312 flexoelectric coefficient component of polyvinylidene fluoride: Deduction, simulation, and mensuration, Sci. Rep. 7, 3134 (2017).

${ }^{29}$ K. Liu, S. Zhang, M. Xu, T. Wu and S. Shen, The research of effective flexoelectric coefficient along 1123 direction in polyvinylidene fluoride, J. Appl. Phys. 121, 174104 (2017).

${ }^{30} \mathrm{~S}$. Baskaran et al., Giant flexoelectricity in polyvinylidene fluoride films, Phys. Lett. A 375, 2082 (2011).

${ }^{31}$ S. Baskaran, X. He, Y. Wang and J. Y. Fu, Strain gradient induced electric polarization in $\alpha$-phase polyvinylidene fluoride films under bending conditions, J. Appl. Phys. 111, 14109 (2012).

${ }^{32} \mathrm{X}$. He, S. Baskaran and J. Y. Fu, On the flexoelectricity in polyvinylidene fluoride films, MRS Online Proc. Libr. Arch. 1403 (2012).

${ }^{33}$ J. Y. Fu, W. Zhu, N. Li and L. E. Cross, Experimental studies of the converse flexoelectric effect induced by inhomogeneous electric field in a barium strontium titanate composition, J. Appl. Phys. 100, 24112 (2006).

${ }^{34}$ V. G. Zalesskii and E. D. Rumyantseva, Converse flexoelectric effect in the $\mathrm{SrTiO}_{3}$ single crystal, Phys. Solid State 56, 1352 (2014).

${ }^{35} \mathrm{~L}$. Shu et al., Converse flexoelectric coefficient $\mathrm{f} 1212$ in bulk $\mathrm{Ba}_{0.67} \mathrm{Sr}_{0.33} \mathrm{TiO}_{3}$, Appl. Phys. Lett. 104, 232902 (2014).

${ }^{36} \mathrm{~L}$. Shu et al., Relationship between direct and converse flexoelectric coefficients, J. Appl. Phys. 116, 144105 (2014).

${ }^{37}$ R. Maranganti, N. D. Sharma and P. Sharma, Electromechanical coupling in nonpiezoelectric materials due to nanoscale nonlocal size effects: Green's function solutions and embedded inclusions, Phys. Rev. B 74, 14110 (2006).

${ }^{38}$ N. D. Sharma, C. M. Landis and P. Sharma, Piezoelectric thin-film superlattices without using piezoelectric materials, J. Appl. Phys. 108, 24304 (2010).

${ }^{39}$ M. S. Majdoub, P. Sharma and T. Cagin, Enhanced size-dependent piezoelectricity and elasticity in nanostructures due to the flexoelectric effect, Phys. Rev. B 77, 125424 (2008).

${ }^{40} \mathrm{~S}$. Shen and S. Hu, A theory of flexoelectricity with surface effect for elastic dielectrics, J. Mech. Phys. Solids 58, 665 (2010).

${ }^{41}$ R. D. Mindlin, Elasticity, piezoelectricity and crystal lattice dynamics, J. Elast. 2, 217 (1972).
${ }^{42}$ A. Abdollahi, C. Peco, D. Millán, M. Arroyo and I. Arias, Computational evaluation of the flexoelectric effect in dielectric solids, J. Appl. Phys. 116, 93502 (2014).

${ }^{43}$ Q. Deng, M. Kammoun, A. Erturk and P. Sharma, Nanoscale flexoelectric energy harvesting, Int. J. Solids Struct. 51, 3218 (2014).

${ }^{44}$ A. Li, S. Zhou and L. Qi, Size-dependent electromechanical coupling behaviors of circular micro-plate due to flexoelectricity, Appl. Phys. A 122, 918 (2016).

${ }^{45} \mathrm{~S}$. Mao and P. K. Purohit, Insights into flexoelectric solids from strain-gradient elasticity, J. Appl. Mech. 81, 81004 (2014).

${ }^{46}$ N. S. Rupa and M. C. Ray, Analysis of flexoelectric response in nanobeams using nonlocal theory of elasticity, Int. J. Mech. Mater. Des. 13, 453 (2017).

${ }^{47}$ J. Hong and D. Vanderbilt, First-principles theory of frozen-ion flexoelectricity, Phys. Rev. B 84, 180101 (2011).

${ }^{48}$ J. Hong and D. Vanderbilt, First-principles theory and calculation of flexoelectricity, Phys. Rev. B 88, 174107 (2013).

${ }^{49} \mathrm{P}$. Hana, Study of flexoelectric phenomenon from direct and from inverse flexoelectric behavior of PMNT ceramic, Ferroelectrics 351, 196 (2007).

${ }^{50} \mathrm{~J}$. Narvaez and G. Catalan, Origin of the enhanced flexoelectricity of relaxor ferroelectrics, Appl. Phys. Lett. 104, 162903 (2014).

${ }^{51}$ D. Lee et al., Giant flexoelectric effect in ferroelectric epitaxial thin films, Phys. Rev. Lett. 107, 57602 (2011).

${ }^{52}$ L. M. Garten, P. Lam, D. Harris, J.-P. Maria and S. TrolierMcKinstry, Residual ferroelectricity in barium strontium titanate thin film tunable dielectrics, J. Appl. Phys. 116, 44104 (2014).

${ }^{53}$ L. M. Garten and S. Trolier-McKinstry, Enhanced flexoelectricity through residual ferroelectricity in barium strontium titanate, $J$. Appl. Phys. 117, 94102 (2015).

${ }^{54}$ L. Shu et al., Flexoelectric behavior in PIN-PMN-PT single crystals over a wide temperature range, Appl. Phys. Lett. 111, 162901 (2017).

${ }^{55}$ L. M. Garten, Residual Ferroelectricity, Piezoelectricity, and Flexoelectricity in Barium Strontium Titanate Tunable Dielectrics (The Pennsylvania State University, 2014).

${ }^{56}$ J. Chen et al., Upward ferroelectric self-polarization induced by compressive epitaxial strain in (001) $\mathrm{BaTiO}_{3}$ films, J. Appl. Phys. 113, 204105 (2013).

${ }^{57}$ L. Shu et al., Frequency dispersion of flexoelectricity in PMN-PT single crystal, AIP Adv. 7, 15010 (2017).

${ }^{58}$ R. Mbarki, J. B. Haskins, A. Kinaci and T. Cagin, Temperature dependence of flexoelectricity in $\mathrm{BaTiO}_{3}$ and $\mathrm{SrTiO}_{3}$ perovskite nanostructures, Phys. Lett. A 378, 2181 (2014).

${ }^{59} \mathrm{~S}$. Huang et al., Flexoelectric characterization of $\mathrm{BaTiO}_{3-0.08} \mathrm{Bi}$ $\left(\mathrm{Zn}_{1 / 2} \mathrm{Ti}_{1 / 2}\right) \mathrm{O}_{3}$, Appl. Phys. Lett. 110, 222904 (2017).

${ }^{60} \mathrm{M}$. A. Beuerlein et al., Current understanding of structureprocessing-property relationships in $\mathrm{BaTiO}_{3}-\mathrm{Bi}(\mathrm{M}) \mathrm{O}_{3}$ dielectrics, J. Am. Ceram. Soc. 99, 2849 (2016).

${ }^{61}$ P. Zubko, G. Catalan and A. K. Tagantsev, Flexoelectric effect in solids, Annu. Rev. Mater. Res. 43, 387 (2013).

${ }^{62}$ J. Narvaez, F. Vasquez-Sancho and G. Catalan, Enhanced flexoelectric-like response in oxide semiconductors, Nature 538, 219 (2016).

${ }^{63} \mathrm{~S}$. R. Kwon et al., Flexoelectricity in barium strontium titanate thin film, Appl. Phys. Lett. 105, 142904 (2014).

${ }^{64} \mathrm{G}$. Catalan et al., Flexoelectric rotation of polarization in ferroelectric thin films, Nature Mater. 10, 963 (2011). 
${ }^{65}$ S. Baskaran, X. He, Q. Chen and J. Y. Fu, Experimental studies on the direct flexoelectric effect in $\alpha$-phase polyvinylidene fluoride films, Appl. Phys. Lett. 98, 242901 (2011).

${ }^{66}$ J. Lu, J. Lv, X. Liang, M. Xu and S. Shen, Improved approach to measure the direct flexoelectric coefficient of bulk polyvinylidene fluoride, J. Appl. Phys. 119, 94104 (2016).

${ }^{67}$ Q. Deng, L. Liu and P. Sharma, Flexoelectricity in soft materials and biological membranes, J. Mech. Phys. Solids 62, 209 (2014).

${ }^{68} \mathrm{M}$. Zelisko et al., Anomalous piezoelectricity in two-dimensional graphene nitride nanosheets, Nat. Commun. 5, 4284 (2014).

${ }^{69}$ K.-A. N. Duerloo, M. T. Ong and E. J. Reed, Intrinsic piezoelectricity in two-dimensional materials, J. Phys. Chem. Lett. 3, 2871 (2012).

${ }^{70}$ A. G. Petrov and L. Mircevova, Is flexoelectricity the coupling factor between chemical energy and osmotic work in the pump? A model of pump, Gen. Physiol. Biophys. 5, 391 (1986).

${ }^{71}$ P.-C. Zhang, A. M. Keleshian and F. Sachs, Voltage-induced membrane movement, Nature 413, 428 (2001).

${ }^{72}$ A. W. Peng, F. T. Salles, B. Pan and A. J. Ricci, Integrating the biophysical and molecular mechanisms of auditory hair cell mechanotransduction, Nat. Commun. 2, 523 (2011).

${ }^{73}$ F. Ahmadpoor and P. Sharma, Flexoelectricity in two-dimensional crystalline and biological membranes, Nanoscale 7, 16555 (2015).

${ }^{74}$ M. Serry and M. A. Sakr, Graphene-metal-semiconductor composite structure for multimodal energy conversion, Sens. Actuators A, Phys. 245, 169 (2016).

${ }^{75}$ W. Zhou, P. Chen, Q. Pan, X. Zhang and B. Chu, Lead-free metamaterials with enormous apparent piezoelectric response, Adv. Mater. 27, 6349 (2015).

${ }^{76}$ X. Zhang, J. Liu, M. Chu and B. Chu, Flexoelectric piezoelectric metamaterials based on the bending of ferroelectric ceramic wafers, Appl. Phys. Lett. 109, 72903 (2016).

${ }^{77}$ W. Zhou and B. Chu, Strong electromechanical response in lead zirconate titanate metamaterials, J. Am. Ceram. Soc. 99, 3317 (2016).

${ }^{78} \mathrm{~W}$. Zhou and B. Chu, Sodium bismuth titanate-based lead-free RAINBOW piezoelectric devices, J. Eur. Ceram. Soc. 37, 2373 (2017).

${ }^{79}$ B. Chu, W. Zhu, N. Li and L. E. Cross, Flexure mode flexoelectric piezoelectric composites, J. Appl. Phys. 106, 104109 (2009).
${ }^{80} \mathrm{M}$. Wan et al., Design of a flexure composite with large flexoelectricity, J. Mater. Sci., Mater. Electron. 28, 6505 (2017).

${ }^{81}$ X. Jiang, W. Huang and S. Zhang, Flexoelectric nano-generator: Materials, structures and devices, Nano Energy 2, 1079 (2013).

${ }^{82}$ W. Huang, S. Yang, N. Zhang, F.-G. Yuan and X. Jiang, Cracks monitoring and characterization by $\mathrm{Ba}_{0.64} \mathrm{Sr}_{0.36} \mathrm{TiO}_{3}$ flexoelectric strain gradient sensors, in Sensors and Smart Structures Technologies for Civil, Mechanical, and Aerospace Systems 2014, Vol. 9061, 906119 (International Society for Optics and Photonics, 2014).

${ }^{83} \mathrm{X}$. Yan et al., A sensor for the direct measurement of curvature based on flexoelectricity, Smart Mater. Struct. 22, 85016 (2013).

${ }^{84} \mathrm{~W}$. Huang et al., Flexoelectric strain gradient detection using $\mathrm{Ba}_{0.64} \mathrm{Sr}_{0.36} \mathrm{TiO}_{3}$ for sensing, Appl. Phys. Lett. 101, 252903 (2012).

${ }^{85}$ S. R. Kwon, W. B. Huang, S. J. Zhang, F. G. Yuan and X. N. Jiang, Flexoelectric sensing using a multilayered barium strontium titanate structure, Smart Mater. Struct. 22, 115017 (2013).

${ }^{86}$ S. D. Hu, H. Li and H. S. Tzou, Distributed flexoelectric structural sensing: Theory and experiment, J. Sound Vib. 348, 126 (2015).

${ }^{87}$ S. R. Kwon, W. B. Huang, S. J. Zhang, F. G. Yuan and X. N. Jiang, Study on a flexoelectric microphone using barium strontium titanate, J. Micromech. Microeng. 26, 45001 (2016).

${ }^{88}$ S. Zhang, K. Liu, M. Xu and S. Shen, A curved resonant flexoelectric actuator, Appl. Phys. Lett. 111, 82904 (2017).

${ }^{89} \mathrm{~S}$. D. Hu, H. Li and H. S. Tzou, Static nano-control of cantilever beams using the inverse flexoelectric effect, in Proc. ASME 2011 International Mechanical Engineering Congress \& Exposition (2011).

${ }^{90} \mathrm{U}$. K. Bhaskar et al., A flexoelectric microelectromechanical system on silicon, Nat. Nanotechnol. 11, 263 (2016).

${ }^{91}$ T. Kim, W. Huang, S. Huang and X. Jiang, Thermal gradient induced flexoelectric effects in bulk $\mathrm{Ba}_{0.67} \mathrm{Sr}_{0.33} \mathrm{TiO}_{3}$, Appl. Phys. Lett. 108, 192902 (2016).

${ }^{92}$ T. Hu, W. Yang, X. Liang and S. Shen, Wave propagation in flexoelectric microstructured solids, J. Elast.1 (2017).

${ }^{93}$ F. Jiao, P. Wei and Y. Li, Wave propagation through a flexoelectric piezoelectric slab sandwiched by two piezoelectric half-spaces, Ultrasonics 82, 217 (2018). 